\title{
Effect of Humidity on The Fatigue Behaviour of Adhesively Bonded Aluminium Joints
}

\section{Abstract}

The present work focuses on the effects of water degradation on the fatigue behaviour of adhesive joints bonded with aluminium adherends. The objective of this study is to measure the influence that humidity has on the fatigue crack growth velocity of two distinct adhesives characterized using the Paris Law, using double cantilever beam (DCB) specimens in unaged and various aged conditions loaded in mode I in order to understand the influence that water content has on the Paris Law constants. It was found that the slope of the Paris Law curve is not heavily changed with the presence of water, but a shift in the curves does occur, generally resulting in a crack initiating at a lower threshold than in the unaged adhesive. Based on this behaviour, it can be concluded that an increase in water content reduces the fatigue joint strength and lifespan of adhesive joints bonded with the studied adhesives.

\section{Keywords}

fatigue, environment, humidity, Paris Law.
M. Costa ${ }^{a^{*}}$

G. Viana ${ }^{\text {a }}$

L. F. M. da Silva ${ }^{b}$

R. D. S. G. Campilho ${ }^{\mathrm{c}}$

${ }^{a}$ INEGI, Rua Dr. Roberto Frias 400, 4200-465 Oporto, Portugal

${ }^{\mathrm{b}}$ DEM, FEUP, Rua Dr. Roberto Frias

s/n, 4200-465 Oporto, Portugal

${ }^{\mathrm{c}}$ DEM, ISEP-IPP, Rua Dr. António Bernardino de Almeida 431, 4200-072

Oporto, Portugal

* Author e-mail: marcelo.costa@fe.up.pt

http://dx.doi.org/10.1590/1679-78252976

Received 08.04.2016

Accepted 16.11.2016

Available online 29.11.2016

\section{INTRODUCTION}

Adhesion technology is increasingly more studied and implemented in various industries, such as the automotive and aeronautical, in order to solve some problems related to the traditional methods of bonding (bolting, riveting, welding, and others). For the automotive industry, adhesives are increasingly more applied both in the assembly of supplementary elements (inside cladding, rubber joints, windows and windscreens) as in structural applications (Pizzi and Mittal 2003). These adhesives are then subjected to humidity due to the various places vehicles may exist in, and are also subjected to fatigue solicitations (driving through holes, hills, even driving in a simple road will cyclically load the structure of a car). Aeronautical joints were the pioneers of adhesive bonding, where both structural and sealing capacities are desired, each with distinct stiffness and mechanical requirements, resulting 
in incredible structural efficiency and durability present in some aircrafts that could not have been achieved with conventional riveted structures (Hart-Smith 2011). Airplanes experience environments with distinct humidity conditions, and are also subjected to cyclic loads (with higher intensity at take-off and landing, but also during flight). Both these industries, and others, are not only concerned with adhesion effectiveness but also with weight efficient structures, which are essential for optimising fuel consumption. As such, aluminium and composites components are often employed and bonded with adhesive, therefore achieving maximum weight reduction. The growing availability of a variety of new materials and significant advances in bonding technology have allowed engineers to trust adhesive joints as a viable alternative to other joining techniques. Because of this, the study and understanding of adhesives has never been more pertinent.

Expanding on the humidity and fatigue remarks made before, adhesive joints are, in many situations and industries, exposed to severe environment conditions during their lifetime that will weaken the adhesive properties as well as the bond strength, thus losing the ability to maintain a good adhesion (Katnam et al. 2010). For both aeronautical and automotive applications, the majority of adhesive-bonded components are exposed to moist air, with varying humidity and temperature throughout the year and location. When the relative humidity $(\mathrm{RH})$ is high over a period of time, the strength and fatigue performance of the joint will gradually decline (Wang et al. 2013). Furthermore, environmental conditions may have an indirect impact on an adhesive joint, for example if they influence the adherend (by thermal expansion of a metal adherend or humidity infiltration in a composite adherend) or the interface between adherend and adhesive.

If we focus on humidity, water diffusion on to an adhesive joint can happen through the adhesive, the adhesive-adherend interface and by cracks or flaws in the adhesive. There are some phenomena responsible for the water degradation of a joint, which are: plasticisation, swelling, hydrolysis or cracking of the adhesive, degradation or change of the interface resulting in loss of adhesion, and corrosion of the substrate. Even though water can negatively affect the adhesive properties, the main factor in the weakening of joints is the water attack on the interface, which causes the most damage and leads to degradation and loss of adhesion between the adhesive and the adherends. The characterisation of humidity's influence on an adhesive is made using Fick's law, which represents the mass gain of a specimen as a function of submersion time, and as a result two parameters can be defined: the diffusion coefficient (related to the speed of water penetration) and the mass at equilibrium (the final mass of the specimen when total water saturation is achieved). Adhesives can exhibit two distinct Fickian behaviours: single Fick and dual Fick. Single Fick is represented by a linear initial behaviour (whose slope is the diffusion coefficient) that eventually stabilizes when saturation is achieved, unlike dual Fick adhesives which have two consecutive and distinct linear stages (therefore they have two diffusion coefficients) and only after the second stage is saturation achieved. In theory, specimen thickness should not influence the behaviour of water or the parameters of Fick's law, a notion postulated by Fujita (1961) who suggested that sorption with different film thicknesses superimpose on a plot of $M_{t} / M_{\infty}$ against $\sqrt{h} / e$ (where $M_{t} / M_{\infty}$ is the mass gain, $\sqrt{h}$ is the square root of submersion time and $e$ is the film thickness), but recent studies have found that adhesives which exhibit dual Fick behaviour shift to single Fick with an increase in specimen thickness (Loh et al. 2005). Temperature is also known to affect the Fickian behaviour of an adhesive, as an increase in temperature leads to an higher coefficient of diffusion, although the mass at equilibrium remaining the same (Rodrigues 
et al. 2016. Submitted). This is useful to reach saturation faster in experimental tests, although care must be taken so the test temperature is well below the glass transition temperature $\left(T_{g}\right)$ of the adhesive.

As mentioned before, aluminium structures are very interesting for structural applications, but the interface between aluminium-adhesive is severely weakened when subjected to moisture. To counteract this effect, surface treatments such as acid etching and phosphoric acid anodising are mandatory for structural joints that rely on aluminium components. Interestingly, the pioneering industries in the use of adhesively bonded weight efficient structures using aluminium joints were the ones that developed surface treatments, notably Boeing who patented the phosphoric acid anodising (PAA) treatment in 1978 (Marceau et al. 1978). The issue of bonding adhesives to aluminium adherends gave rise to various types of surface treatments being investigated and developed, such as chromic acid anodizing (CAA), chromic acid etching (CAE), sulphuric acid anodization (SAA), the aforementioned PAA, and others. Under static conditions the effect of the surface treatment is not extremely felt, for example in the case of a lap shear strength test performed by Briskham and Smith (2000) were the PAA treated joint exhibited a $25 \mathrm{MPa}$ strength compared to $21 \mathrm{MPa}$ by the simply abraded joint. The true benefit has been shown in various works (Briskham and Smith 2000, Lefebvre et al. 2002, Fernando et al. 1996, Kinloch et al. 2000, Abel et al. 2006) to happen under fatigue conditions where PAA joints have proved to be the superior surface treatment for the various conditions studied by the authors. As a result, PAA is the optimal treatment to apply in adhesive bonds subjected to environment, especially those that are subjected to fatigue (Costa et al. 2016. Submitted).

When studying fatigue, two approaches may be taken: establishing S-N curves, where joints are tested under varying stresses and/or environments until failure and plotted in a diagram to determine the expected fatigue life, or Paris Law curves, which measure the crack growth velocity of an adhesive under different environments. Both are useful and have consequently been studied by various authors, although not as much as would be expected (Costa et al. 2016. Submitted). As a result, results exist but do not always paint a clear picture on what happens to an aged joint (Costa et al. 2016. Submitted).

Paris Law, which is the fatigue approach studied in this work, consists on the cyclical solicitation of a joint, which is then subjected to periodic measurements of crack length. Simply put, this leads to information on the various crack dimensions at specific cycles, which is then used to obtain the variation of the crack length through the entire test. How that variation is obtained depends on the method selected, and ASTM E647 (2000)defines two: the secant method and the polynomial method. With this information the Paris Law may be obtained, as is shown in Figure 1.

Paris Law consists of three regions: the first (Zone 1) where crack initiation occurs, the second (Zone 2) where stable crack propagation happens, and lastly Zone 3 where failure of the joint takes place. Zone 2 is the key area, where a line (after logarithmic axis are applied) can be obtained and two constants obtained: $m$, the slope, and $C$, the intersection of the line with the y-axis. A bigger value for $m$ (maintaining $C$ ) would translate in a crack that propagates more quickly, while a higher $C$ (maintaining $m$ ) means that the crack propagates by a larger length each time, although at the same speed.

Although studying fatigue, the static mechanical properties are also important. They need to be obtained to define the fatigue loading (for example, maximum fatigue load is $60 \%$ of maximum static 
load), and also are needed to help justify the fatigue differences between aged stages (for example, if the ductility increases a lot from $40 \%$ to $60 \% \mathrm{RH}$, we may expect that the crack propagation speed decreases for those same stages) and between different adhesives.

In the present work, humidity's influence on the fatigue properties of two adhesives is experimentally investigated, including the mass gain due to water and the effect on the Paris Law curves and corresponding constants.

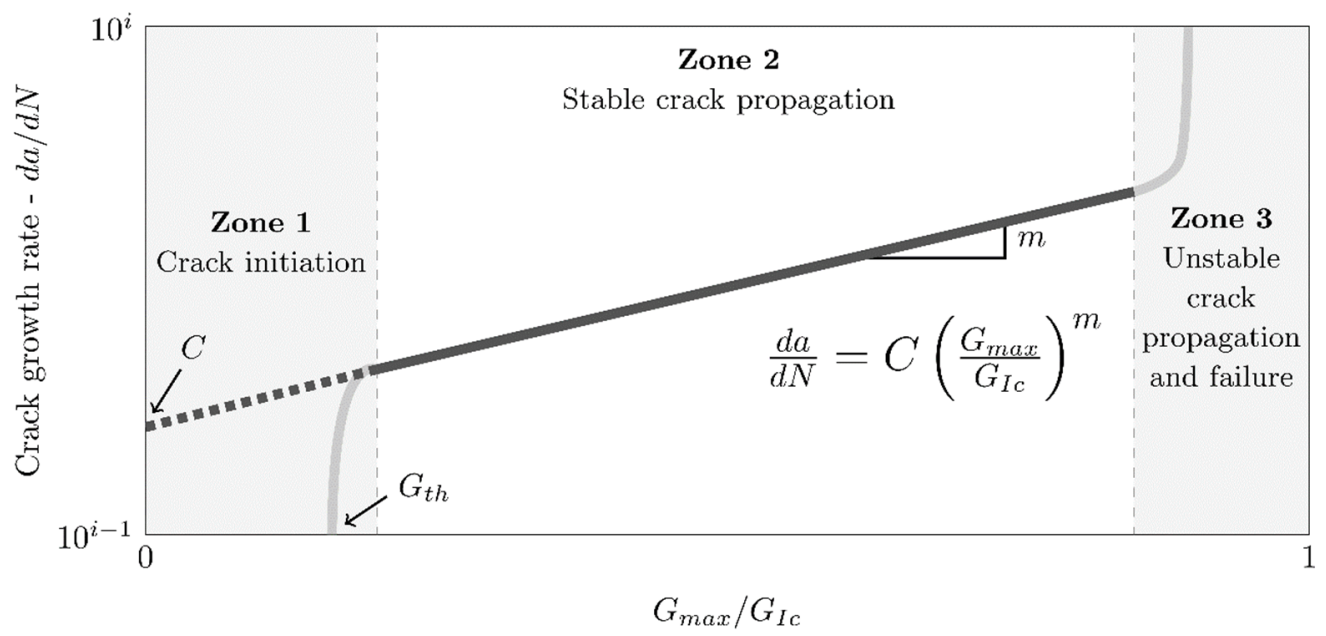

Figure 1: Representation of the Paris Law for adhesive joints.

\section{EXPERIMENTAL DETAILS}

\subsection{Adhesive Characterization}

Two adhesives with distinct properties were studied with the objective of determining the fatigue behaviour degradation due to humidity for distinct conditions. The first epoxy adhesive, XNR 68521, supplied by NAGASE CHEMTEX@ (Osaka, Japan), is a one-part system that cures at $150{ }^{\circ} \mathrm{C}$ for 3 hours. It has a linear structure, which allows greater freedom of movement to the chains, unlike the cross-linked structure of a conventional epoxy adhesive. As a consequence, this polymer has some features of thermoplastic polymers due to the resulting linear structure. The second adhesive is SikaPower 4720, supplied by SIKA@ (Vila Nova de Gaia, Portugal). The tensile properties of each adhesive and the fracture toughness $\left(G_{I C}\right.$ have been determined in a previous work (Costa et al. 2016) and are reproduced in Table 1.

\begin{tabular}{ccccc}
\hline \hline Adhesive & $\begin{array}{c}\text { Young's Modulus } \\
(\mathrm{MPa})\end{array}$ & $\begin{array}{c}\text { Tensile Strength } \\
(\mathrm{MPa})\end{array}$ & $\begin{array}{c}\text { Strain at } \\
\text { failure }(\%)\end{array}$ & $G_{I C}(\mathrm{~N} / \mathrm{mm})$ \\
\hline NagaseXNR 6852-1 & $2095 \pm 120.8$ & $61.88 \pm 1.64$ & $15.99 \pm 7.66$ & $5.89 \pm 0.62$ \\
SikaPower 4750 & $2030.9 \pm 86.7$ & $31.41 \pm 1.41$ & $3.45 \pm 0.55$ & $1.58 \pm 0.18$ \\
\hline \hline
\end{tabular}

Table 1: -Mechanical properties of each adhesive for the unaged state (Costa et al. 2016). 
Moisture absorption tests have also been performed to determine the behaviour of each adhesive when submerged in water (Costa et al. 2016), which was modelled using Fick's law. The diffusion coefficient $(D)$ and mass at equilibrium $\left(M_{\infty}\right)$ for each adhesive are reproduced in Table 2.

\begin{tabular}{ccc}
\hline \hline Adhesive & $\begin{array}{c}\text { Diffusion coefficient } \\
\left(\mathrm{m}^{2} / \mathrm{s}\right)\end{array}$ & $\begin{array}{c}\text { Mass at equilibrium } \\
(\%)\end{array}$ \\
\hline Nagase XNR 6852-1 & $5.02 \times 10^{-13}$ & 1.3 \\
SikaPower 4750 & $0.89 \times 10^{-13}$ & 32.5 \\
\hline \hline
\end{tabular}

Table 2: Fick's law coefficients for both studied adhesives (Costa et al. 2016).

The remaining needed adhesive information is how they degrade when in contact with water. The fatigue behaviour will be studied and presented in this paper, but the static behaviour will also be necessary to aid in the discussion of results. The degradation of each mechanical property presented in Table 1 has been determined (Costa et al. 2016), and a handy formula was established to find the value of the property as a function of the relative humidity content. The formula for Young's modulus, tensile strength and strain is the following:

$$
y(R H)=\left(y_{0}-y_{f}\right) * \exp \left(-\frac{R H}{\left(10^{13} / 2\right) \cdot D \cdot M_{\infty}}\right)+y_{f}
$$

Where $y$ is the mechanical property being predicted, $y_{0}$ is the property value in the unaged stage, $y_{f}$ the value in the fully saturated stage, $R H$ is the percentage value of relative humidity present in the joint $(0 \leq R H \leq 100)$, and both $D$ and $M_{\infty}$ are the Fick's law coefficients already presented in Table 2 for each adhesive. The formula for the fracture toughness is not so simple and as such is not presented here. Nonetheless, the values themselves are available in (Costa et al. 2016) for examination if desired.

\subsection{Specimen Geometry}

To characterise the effects of humidity on the fatigue response of adhesively bonded aluminium joints reduced double cantilever beam (DCB) specimens were used as shown in Figure 2. Themainpurpose of suchspecimenisthereduction of thewidth of theadhesivelayer ( $5 \mathrm{~mm}$ in the case of Figure 2 and 25.4 $\mathrm{mm}$ for the standard DCB specimens (ASTM 1999)) due to the fact that humidity takes a long time to ingress into the adhesive layer, and thus the level of joint saturation (relative humidity) progresses much slower the thicker the width, as illustrated in Figure 3. Reduction of the length of the specimen $(120 \mathrm{~mm})$ is also desirable so that the specimens can be fully immersed in the available flasks and furthermore fit inside the environmental test chamber (so that in future studies temperature may also be studied). Care must be taken so that the reduction of dimensions results in results comparable to those of the standard DCB, and to that effect previous studies have been published (Costa et al. 2015). 
a)
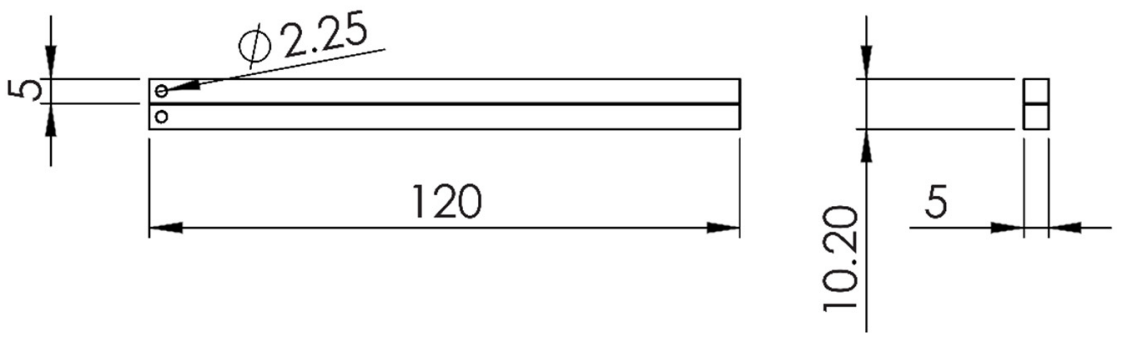

b)
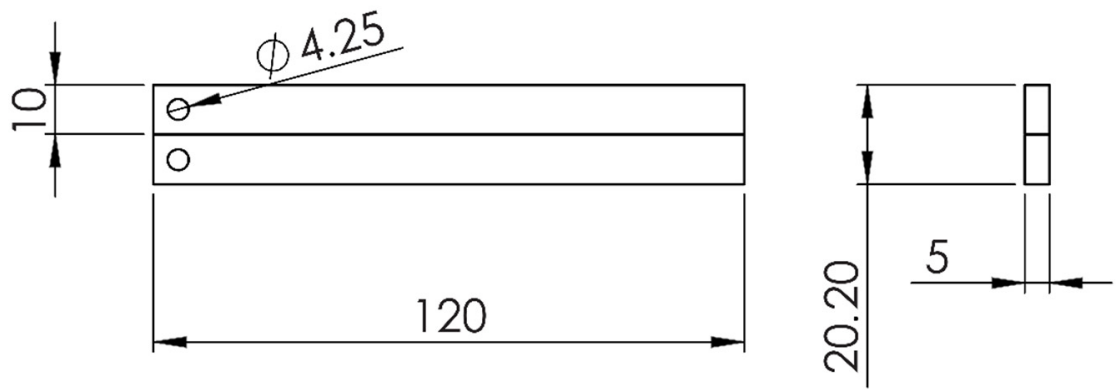

Figure 2: Reduced DCB specimen dimensions used for mode I tests: a) specimen used with the SikaPower 4720 adhesive, b) specimen used with the Nagase XNR 6852-1 adhesive (dimensions in mm).

Figure 2 shows two identical types of reduced DCB specimens, the only difference between them being substrate thickness (in one case $5 \mathrm{~mm}$, and in the other $10 \mathrm{~mm}$ ). This is the case because a preliminary optimised specimen (Figure 2 a)) was defined under the assumption of $G_{I c} \cong 2 \mathrm{~N} / \mathrm{mm}$, which was based on the fact that both SikaPower 4720 and Nagase XNR 6852 (the previous version of the adhesive studied in this work - note the absence of "- 1 " in the designation) had a $G_{I C}$ inferior or around $2 \mathrm{~N} / \mathrm{mm}$ (as published in (Saldanha et al. 2013) for Nagase XNR 6852). After specimen manufacturing, immersion and testing it was noticed that while the SikaPower 4720 specimens behaved as expected, Nagase XNR 6852-1 specimens experienced adherend deformation. The fracture toughness tests were performed after this happened, which showed that Nagase XNR 6852-1 had a higher $G_{I c}$ as shown in Table 1 . Because of this a new geometry was defined to avoid plastic deformation of the aluminium adherends, as seen in Figure 2 b). All the Nagase XNR 6852-1 specimens were manufactured according to this geometry, and the $5 \mathrm{~mm}$ thickness geometry was maintained for the SikaPower 4720 adhesive (both because of the much longer time needed to saturate the adhesive, as seen in Figure 3, and the fact that several specimens were already submerged and replacing them all would delay the results for SikaPower 4720 for several months).

\subsection{Manufacture and Testing Procedures}

To obtain the final testable specimen, the following stages had to be followed: application of surface treatment to the aluminum adherends, DCB specimen manufacturing, specimen ageing and finally experimental testing.

The applied surface treatment was phosphoric acid anodizing (PAA), which is the standard surface preparation for aluminum bonds. PAA has positive results under static conditions and excellent results under fatigue conditions (more details can be found in (Costa et al. 2016. Submitted) which reviews various surface treatments for aluminum joints under different conditions). PAA is defined 
by ASTM D3933 (ASTM 2010), and as such the procedure detailed in the standard was followed. After application of the surface treatment, the adherends were kept in a contamination-free environment and used for specimen manufacturing between 8 to 24 hours after PAA application. DCB specimen manufacturing was conducted in a custom aluminum mold (to account for the thermal expansion of the aluminum if curing at high temperature and avoid the creation of residual stresses in the joints) and conducted at the temperature and during the time required by each adhesive in a INTOCO $\mathbb{R}$ (Staverton, United Kingdom) hot-plates press under $2 \mathrm{MPa}$ hydrostatic pressure. After manufacturing the excess adhesive was removed. Specimen ageing was performed under full immersion in distilled water at $32{ }^{\circ} \mathrm{C}$ in a MMM@) (Munich, Germany) Friocell environmental chamber. Experimental testing was performed in a MTSR) (Minnesota, USA) servo-hydraulic machine under constant load control.

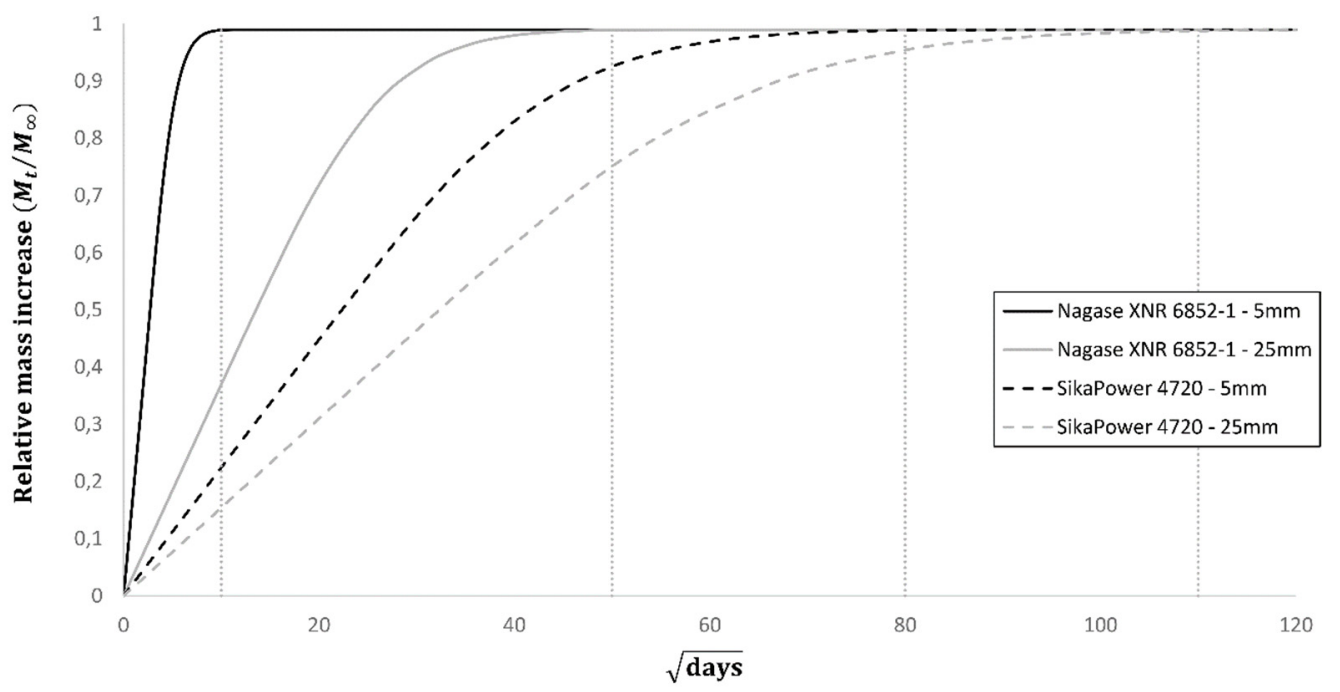

Figure 3: Comparison between saturation times for $5 \mathrm{~mm}$ and $25 \mathrm{~mm}$ thicknesses using both adhesives.

Three specimens were first tested under static conditions to obtain the fracture toughness of the adhesive (results published in (Costa et al. 2016)). A side result of such tests was the static failure load (for each adhesive and aged state), which was then used to determine the fatigue parameters. The maximum fatigue load was set to $60 \%$ of the static failure load, the frequency was $1 \mathrm{~Hz}$ and a ratio of 0.1 was imposed. At least 5 specimens were then tested under fatigue, with the objective of confirming the behavior beyond doubt (fatigue results have a certain associated uncertainty, which could be worsened with) and guaranteeing no further tests are needed (which would require new specimen manufacturing and as such a long time to obtain new results). In each test the applied load and measured displacement were recorded for each cycle. The crack length was obtained using the Compliance-based beam method (CBBM) (de Moura et al. 2008, Fernández et al. 2011), which uses the specimen compliance (determined using the recorded load and displacement) and has the advantage of making it possible to deduce an equivalent crack length through the application of Timoshenko's beam theory, eliminating the need to constantly measure the crack visually and the inevitable visual errors. 
Figure 4 shows the raw result of a fatigue test, in this case under a constant load of $120 \mathrm{~N}$, and respective data treatment. Regarding the load-displacement plot, there are three regions: the first part, between 0 and approximately 100 cycles, where the load has not yet, the second section where the load is maintained at the target value and the displacement can be seen to steadily progress in a controlled manner, and finally a third region where unsteady crack growth happens and as such the load can no longer be maintained and the displacement increases abruptly until specimen failure. Through the CBBM method the raw load-displacement data can be used to obtain the equivalent crack length, as pictured in Figure 4 (bottom left), and the maximum strain energy release rate $\left(G_{m a x}\right)$, therefore resulting in all the necessary data to calculate the Paris Law (Figure 4 bottom right). ASTM E647 (ASTM 2000) defines two methods for obtaining the Paris Law: secant method and incremental polynomial method. The latter is more useful for visual measures of the crack length during testing, which will naturally have some errors and as such the method smooths out such errors. The secant method is simpler and, because CBBM already calculates an equivalent crack length, such smoothing is inherent to CBBM and as such the secant method was used in this work.
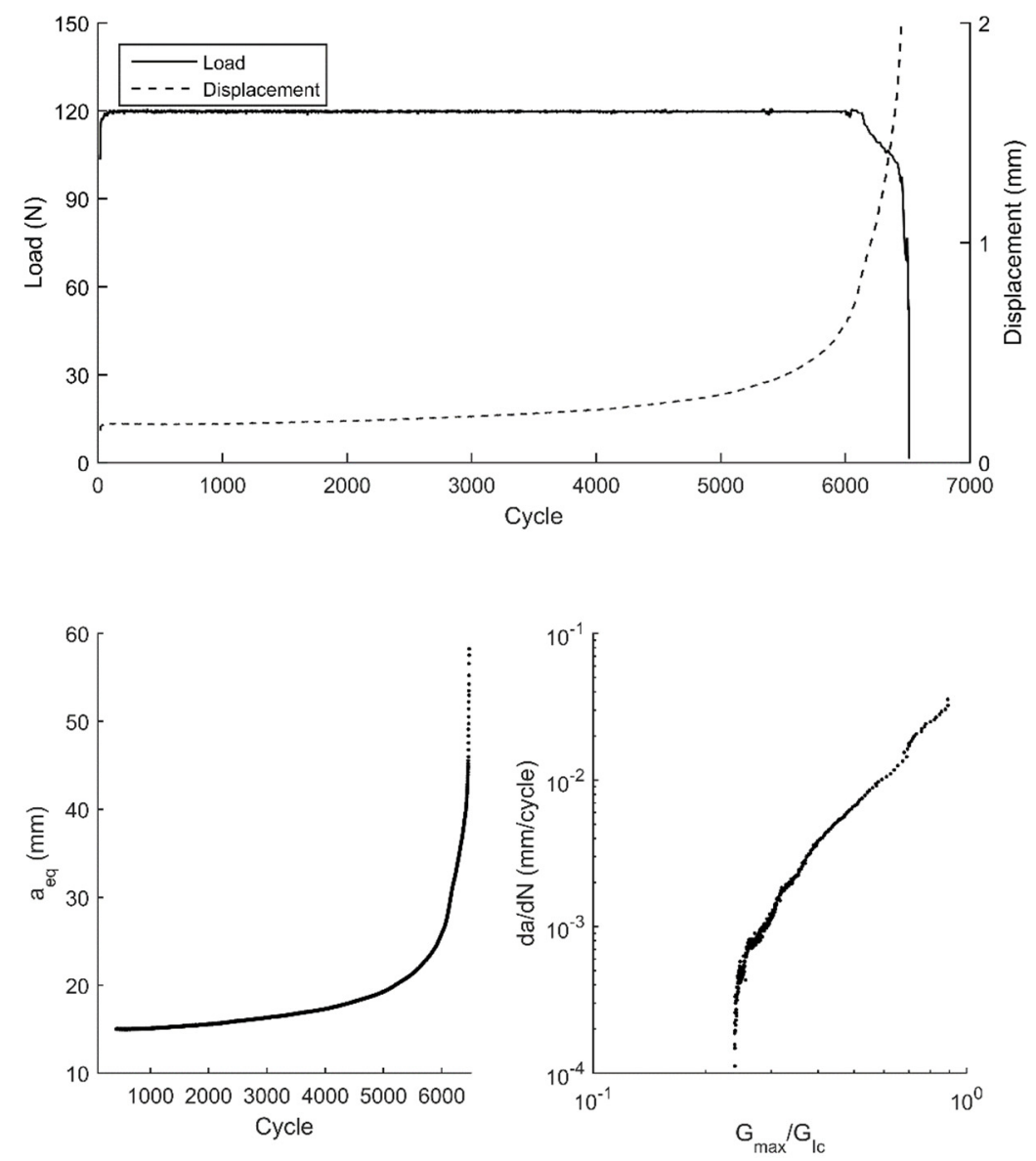

Figure 4: Example of experimental fatigue test: raw load-displacement data direct from machine (top), equivalent crack length as a function of cycles (bottom left), and respective Paris Law representation (bottom right). 


\section{EXPERIMENTAL RESULTS AND DISCUSSION}

\subsection{Crack Growth Velocity}

Based on the previously presented adhesive moisture absorption data, DCB specimens were taken out of water at specific instances chosen such that the average relative humidity through the joint represented specific values of saturation such as $25 \% \mathrm{RH}, 40 \%$, etc. The results of the unaged state compared with three aged states for both adhesives are presented in Figure 5.
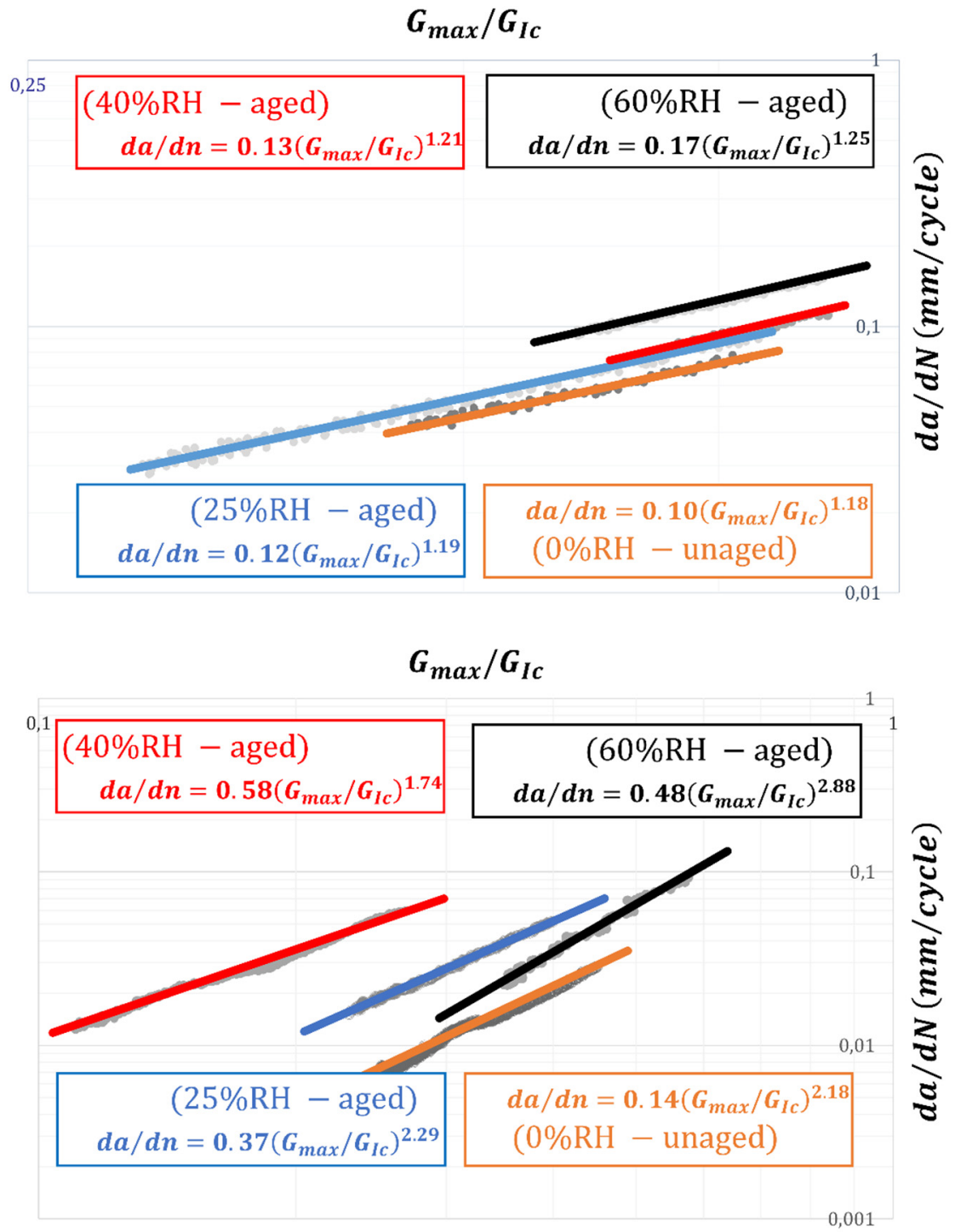

Figure 5: Crack growth velocity as a function of relative humidity:

Nagase XNR 6852-1 (top), SikaPower 4720 (bottom). 
For the Nagase XNR 6852-1 adhesive, the results are within what was expected as it is the behaviour usually reported in the literature: an increase of relative humidity translates to a shift in the upper direction of the stable crack propagation part of the Paris Law, which implies that the crack propagates at the same velocity (slope of the line, $m$, is more or less constant) but starts propagating sooner $\left(G_{t h}\right.$ appears to decrease with an increase of $\mathrm{RH}$, meaning the crack initiates propagation sooner). Specifically regarding the slope, it can be seen that although visually it appears constant, in reality the values in the equation increase with an increase of $\mathrm{RH}$, although very slightly. This makes sense if we consider that the diffusion behaviour of Nagase XNR 6852-1 consists on low mass gain due to water absorption (fully saturates at 1.3\%), and thus such a small variation of the slope is not taken into account.

Regarding SikaPower 4720 the situation is not as clear. Although up to $40 \% \mathrm{RH}$ the behaviour is consistent with the previously exposed notion that an increase in relative humidity shifts the Paris Law upward, for $60 \% \mathrm{RH}$ the line shifted to the right and the slope increased, effectively signifying that the propagation occurs at a higher velocity (expected), and that the crack starts to propagate at a later $G_{t h}$ (which would mean a $60 \% \mathrm{RH}$ is good to delay crack initiation). This last detail is contradictory with all the previous results, but may be explained due to the huge mass gain experienced by SikaPower 4720 when in contact with water (a $32.5 \%$ increase in mass when fully saturated). SikaPower 4720 experiences, when fully saturated, prominent swelling when compared with Nagase XNR 6852-1, as visible in Figure 6, and also attains a rubbery behaviour when handled manually unlike in the unaged state.

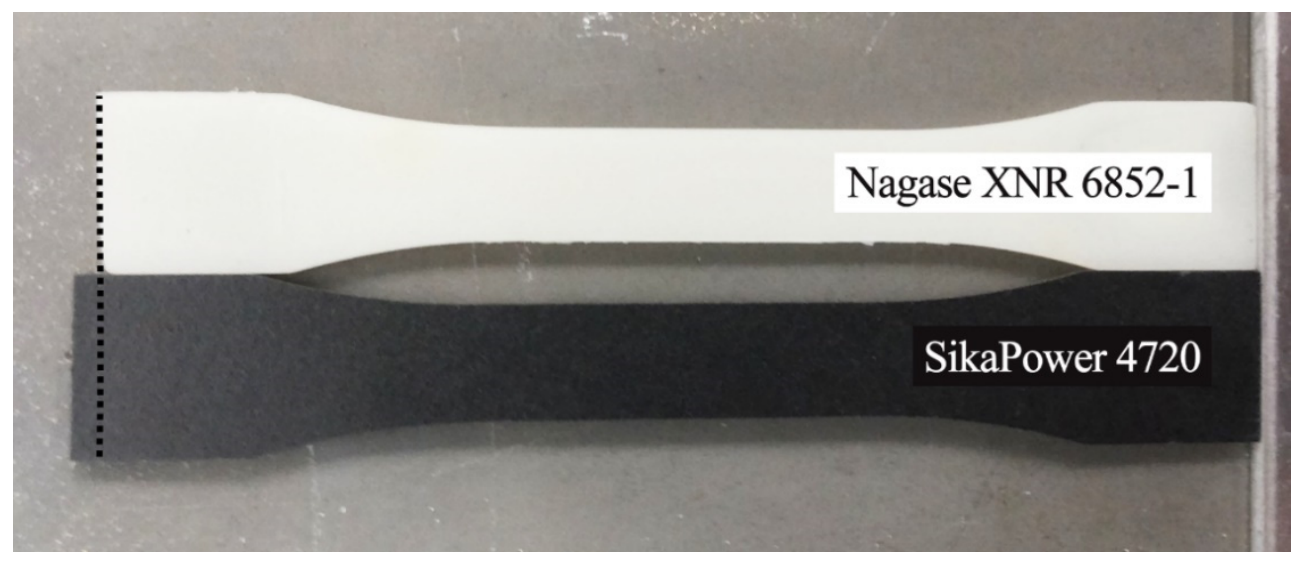

Figure 6: Swelling comparison of both adhesives in the saturated stage.

Such swelling and changes in physical behaviour (rubbery state) may imply hydrolysis and chemical degradation of the adhesive, which explains why the crack propagation velocity is higher. Regarding a later $G_{t h}$ than expected, the swelling and increase in ductility (when compared to the unaged and early aged stages) may contribute to extra microscopic obstruction to crack initiation, such as wider voids and water particles absorbing energy, therefore delaying crack initiation. Finally, the glass transition temperature $\left(T_{g}\right)$ of the adhesives in the saturated stage (Viana et al. 2016. Submitted) may offer the final explanation: while in the unaged stage Nagase XNR 6852-1 and SikaPower 4720 
have a $T_{g}$ of $120{ }^{\circ} \mathrm{C}$ and $100{ }^{\circ} \mathrm{C}$ respectively, in the saturated stage Nagase XNR 6852-1 drops to $100{ }^{\circ} \mathrm{C}$ and SikaPower 4720 drops to below room temperature. This has two major consequences: first that there is major degradation of the adhesive due to water, and secondly that the drop from $100{ }^{\circ} \mathrm{C}$ at $0 \% \mathrm{RH}$ to below room temperature at $100 \%$ implies that at some stage in-between $0 \%$ and $100 \%$ $\mathrm{RH}$ we begin testing the adhesive at a temperature above its $T_{g}$. It is estimated that at around $40 \%$ RH the $T_{g}$ of SikaPower 4720 reaches the ageing temperature and therefore will have its physical properties changed completely, which would certainly explain the change in fatigue behaviour of SikaPower 4720 after that relative humidity value.

Comparing between both adhesives further interesting aspects arise: we see that Nagase XNR 6852-1 has a much slower crack propagation velocity (an $m$ value of around 1.2 when compared to SikaPower which is roughly double), and combining that with the evidence that it absorbs much less water (Table 2), has its mechanical properties barely degraded when in contact with water (Costa et al. 2016), and its fatigue behaviour is more predictable and consistent (Figure 5) makes Nagase XNR 6852-1 an appealing adhesive for long-term applications.

\subsection{Prediction of Paris Law Constants}

To further compare the fatigue behaviour between both adhesives and in an effort to develop prediction tools for such behaviour, the slope $(m)$ and y-axis intersection $(C)$ values of the Paris Law for all the presented situations was analysed. The behaviour of each variable appears to grow in a quadratic manner with an increase in relative humidity, and as such the following prediction equation was postulated:

$$
\operatorname{var}(R H)=K \cdot R H^{2}+\operatorname{var}_{0}
$$

The idea of Equation 2 is that it is valid for both Paris Law variables and also for both adhesives, and as such the constant $K$ must be determined as dependent on both adhesive properties. Following the same notion as presented in Equation (1) and used in other works (Costa et al. 2016), we postulated that the Fick's Law parameters should be essential to the prediction, and have arrived at the following formulation for $K$ :

$$
K=\frac{2}{3} \cdot 10^{8} \cdot D \cdot M_{\infty}
$$

By combining Equation 3 with 2 and applying it to both Paris Law parameters results in the behaviour shown in Figure 7 (top and middle), where the experimental points are also overlaid to aid the comparison with the predicted values. The $T_{g}$ of both adhesives as a function of relative humidity is also presented in Figure 7 (bottom), with further details about the method and results published elsewhere (Viana et al. 2016. Submitted). Two critical areas are shown in the $T_{g}$ results, which are confined by the temperatures $20^{\circ} \mathrm{C}$ and $32.5^{\circ} \mathrm{C}$, representing room temperature and ageing temperature, respectively, because when the $T_{g}$ decreases below such temperatures a drastic change in properties and response may be expected. 

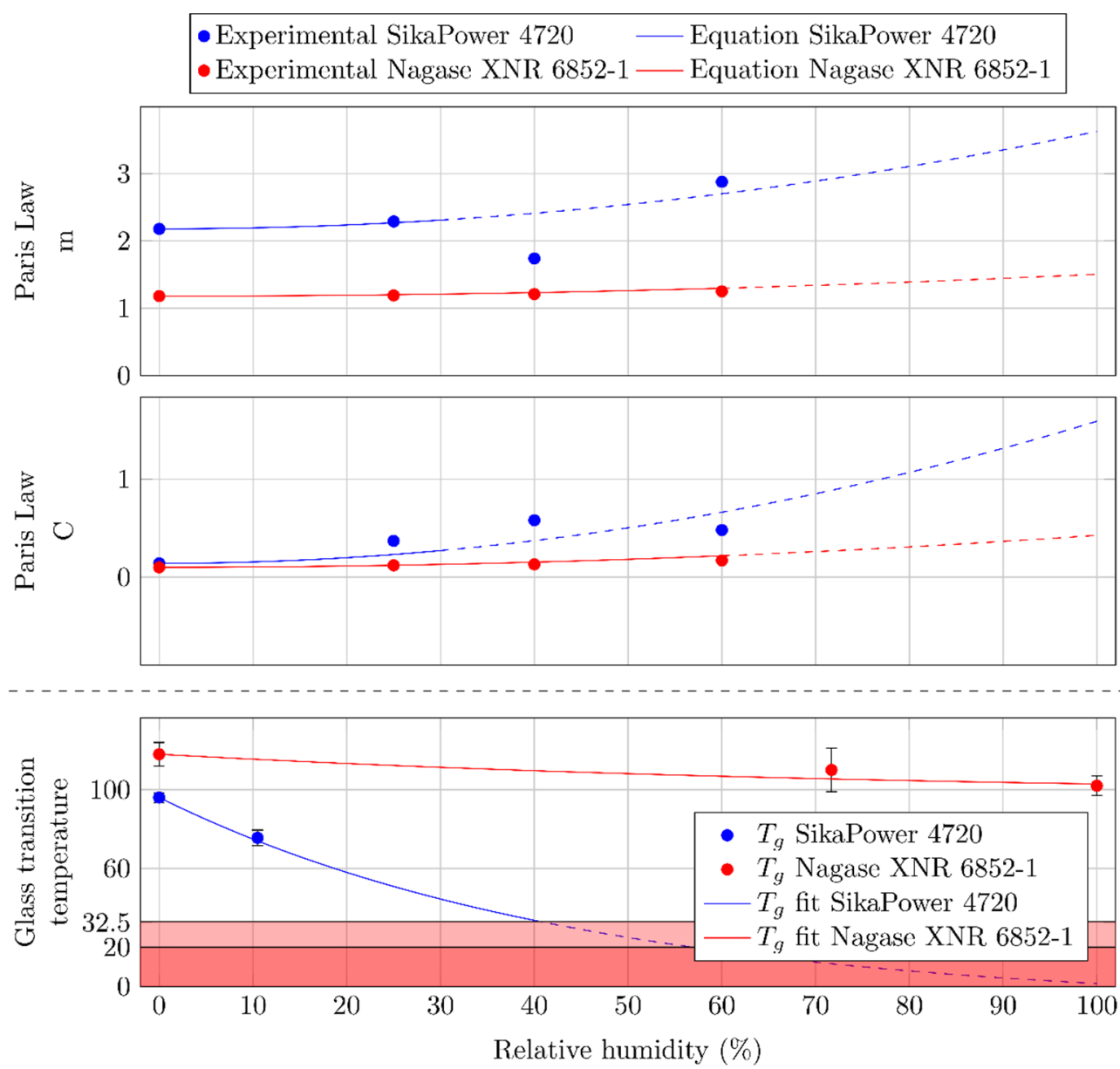

Figure 7: Experimental Paris Law values for both adhesives as a function of relative humidity overlaid on the predicted values obtained using Equation 2 and 3 (top and middle), $T_{g}$ of both adhesives as a function of relative humidity (Viana et al. 2016. Submitted) (bottom).

Various interesting effects are now easier to visualize: there is a clear shift away from the expected values for SikaPower 4720 after $40 \% \mathrm{RH}$, a behaviour consistent with the fact that its $T_{g}$ fall below ageing temperature at that point, while Nagase XNR 6852-1 behaves precisely as expected due to its $T_{g}$ being comfortably above the ageing and testing temperature. Another remark is that the exact same equation accurately predicts the behaviour of both Paris Law parameters for both adhesives (while operating below $T_{g}$ ) through the determination of a universal constant (Equation 3) that is dependent on the Fick's Law parameters of each adhesive. Finally, we can infer the future Paris Law values at a higher relative humidity content due to the good correlation between experimental and predicted values. In future works this will be confirmed, especially for SikaPower 4720 where it will be interesting to see how the behaviour evolves due to it being tested above its $T_{g}$, and also see how this prediction equation applies to other adhesives. 


\section{CONCLUSIONS}

Two epoxy adhesives had their fatigue response studied due to varying degrees of water content, which resulted in crack growth curves that evolve in a predictable manner when the relative humidity content changes. Nagase XNR 6852-1 was expected to experience small changes in the crack growth curves with increasing humidity due to its very stable behaviour when submerged in water, which was confirmed in the experimental Paris Law curves. SikaPower 4720 was expected to have its fatigue behaviour change in a more accentuated manner due to its much larger water absorption parameters (32.5\% mass increase when saturated compared to 1.2\% for Nagase XNR 6852-1, an increase of over 27 times), which also happened, but the behaviour was not as predictable as Nagase XNR 6852-1 due to the fact that the $T_{g}$ of SikaPower 4720 decreases drastically with an increase of relative humidity. Based on the experimental data, an equation is proposed which fits the behaviour of both adhesives (while they are away from $T_{g}$ ) and produces results very close to the experimental values. In the future the authors hope to apply this prediction to other adhesives.

The study of changes in the fatigue response on adhesively bonded joints due to varying degrees of relative humidity is an important step in understanding how joints will behave in the long-term, and although there is already some data on the subject it is not enough to draw clear conclusions (Costa et al. 2016. Submitted), which the authors believe this work tries to help in.

\section{Acknowledgments}

The authors would like to thank Fundação para a Ciência e a Tecnologia (FCT) for supporting this work through grant EXCL/EMS-PRO/0084/2012, Sika for supplying the SikaPower 4720 adhesive, and Nagase Chemtex for supplying XNR 6852-1 adhesives used in this work.

\section{References}

Abel, M.-L., Adams, A., Kinloch, A., Shaw, S.,Watts, J. (2006). The effects of surface pretreatment on the cyclicfatigue characteristics of bonded aluminium-alloy joints. International journal of adhesion and adhesives 26:50-61.

ASTM (1999). D3433 - Test Method for Fracture Strength in Cleavage of Adhesives in Bonded Metal Joints

ASTM (2000). E647 - Standard Test Method for Measurement of Fatigue Crack Growth Rates

ASTM (2010). D3933 - Standard Guide for Preparation of Aluminum Surfaces for Structural Adhesives Bonding (Phosphoric Acid Anodizing)

Briskham, P.,Smith, G. (2000). Cyclic stress durability testing of lap shear joints exposed to hot-wet conditions. International journal of adhesion and adhesives 20:33-38.

Costa, M., Viana, G., Canto, C., et al. (2015). Effect of the size reduction on the bulk tensile and double cantilever beam specimens used in cohesive zone models. Proceedings of the Institution of Mechanical Engineers, Part L: Journal of Materials Design and Applications.

Costa, M., Viana, G., da Silva, L. F. M.,Campilho, R. D. S. G. (2016). Effect of humidity on the mechanical properties of adhesively bonded aluminium joints. Part L: Journal of Materials: Design and Applications.

Costa, M., Viana, G., da Silva, L. F. M.,Campilho, R. D. S. G. (2016. Submitted). Environmental effect on the fatigue degradation of adhesive joints: a review. The Journal of Adhesion. 
de Moura, M. F. S. F., Campilho, R. D. S. G.,Goncalves, J. P. M. (2008). Crack equivalent concept applied to the fracture characterization of bonded joints under pure mode I loading. Composites Science and Technology 68:22242230 .

Fernández, M., De Moura, M., Da Silva, L.,Marques, A. (2011). Composite bonded joints under mode I fatigue loading. International Journal of Adhesion and Adhesives 31:280-285.

Fernando, M., Harjoprayitno, W.,Kinloch, A. (1996). A fracture mechanics study of the influence of moisture on the fatigue behaviour of adhesively bonded aluminium-alloy joints. International journal of adhesion and adhesives 16:113119.

Fujita, H. (1961). Free-volume model of diffusion in polymer solutions. Advances in Polymer Science 3:1-47.

Hart-Smith, L. J. 2011. Adhesively Bonded Joints in Aircraft Structures. In Handbook of Adhesion Technology, edited by L. M. da Silva, A. Öchsner, and R. Adams: Springer Berlin Heidelberg.

Katnam, K., Sargent, J., Crocombe, A., Khoramishad, H.,Ashcroft, I. (2010). Characterisation of moisture-dependent cohesive zone properties for adhesively bonded joints. Engineering Fracture Mechanics 77:3105-3119.

Kinloch, A., Little, M.,Watts, J. (2000). The role of the interphase in the environmental failure of adhesive joints. Acta Materialia 48:4543-4553.

Lefebvre, D., Ahn, B., Dillard, D.,Dillard, J. (2002). The effect of surface treatments on interfacial fatigue crack initiation in aluminum/epoxy bonds. International journal of fracture 114:191-202.

Loh, W., Crocombe, A., Wahab, M. A.,Ashcroft, I. (2005). Modelling anomalous moisture uptake, swelling and thermal characteristics of a rubber toughened epoxy adhesive. International journal of adhesion and adhesives 25:1-12.

Marceau, J. A., Firminhac, R. H.,Moji, Y. 1978. Method for providing environmentally stable aluminum surfaces for adhesive bonding and product produced. USA: The Boeing Company.

Pizzi, A.,Mittal, K. L. (2003). Handbook of Adhesive Technology, Revised and Expanded, Taylor \& Francis.

Rodrigues, T. A. F., Chaves, F. J. P., da Silva, L. F. M., Costa, M.,Barbosa, A. Q. (2016. Submitted). Determination of the fracture envelope of an adhesive joint as a function moisture. Materialwissenschaft und Werkstofftechnik.

Saldanha, D. F. S., Canto, C., da Silva, L. F. M., et al. (2013). Mechanical characterization of a high elongation and high toughness epoxy adhesive. International Journal of Adhesion and Adhesives 47:91-98.

Viana, G., Costa, M., Banea, M.,da Silva, L. F. M. (2016. Submitted). Behaviour of environmentally degraded epoxy adhesives as a function of temperature. The Journal of Adhesion.

Wang, M., Liu, A., Liu, Z.,Wang, P.-C. (2013). Effect of hot humid environmental exposure on fatigue crack growth of adhesive-bonded aluminum A356 joints. International Journal of Adhesion and Adhesives 40:1-10. 